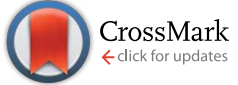

Cite this: J. Mater. Chem. A, 2015, 3, 15119

Received 14th April 2015

Accepted 11th June 2015

DOI: $10.1039 / \mathrm{c} 5 \mathrm{ta0} 2679 \mathrm{~b}$

www.rsc.org/MaterialsA

\section{Chirality analysis of horizontally aligned single- walled carbon nanotubes: decoupling populations and lengths $\dagger$}

\begin{abstract}
Taiki Inoue, ${ }^{a}$ Daisuke Hasegawa, ${ }^{a}$ Shohei Chiashi ${ }^{a}$ and Shigeo Maruyama*ab
Utilizing the aligned morphology of single-walled carbon nanotubes (SWCNTs) grown on crystal quartz substrates together with systematic Raman mapping measurements, the populations and lengths of SWCNTs with different chiralities $(n, m)$ were independently evaluated. Chiralities of SWCNTs were assigned on a one-by-one basis by comparing radial breathing mode frequencies with the Kataura plot. The SWCNT lengths were determined by Raman mapping and/or scanning electron microscopy. Both the populations and lengths of the SWCNTs grown in this study with a diameter of 1.14 to $1.29 \mathrm{~nm}$ showed no clear dependence on their chiral angles.
\end{abstract}

\section{Introduction}

The atomic configurations of single-walled carbon nanotubes (SWCNTs) ${ }^{1}$ are typically represented by two integers $(n, m)$, signifying the chirality, since the electrical and optical properties of SWCNTs differ depending on chirality. ${ }^{2,3}$ While as-grown SWCNT ensembles are generally composed of SWCNTs with various chiralities, specific SWCNTs possessing appropriate properties are required for electrical and optical applications. Although separation techniques to obtain chirality-specific SWCNTs from as-grown samples have been developed, ${ }^{4,5}$ direct growth of chirality-controlled SWCNTs is still highly desirable.

The chiral index $(n, m)$ is equivalent to the pair of the diameter and chiral angle of the SWCNTs. Control of the SWCNT diameter is possible, to a certain extent, via tuning the diameter of catalytic nanoparticles during the chemical vapour deposition (CVD) growth process. ${ }^{6}$ Various growth conditions and characterization methods have demonstrated the chiralitycontrolled growth of SWCNTs and have shown that the abundance of chiral SWCNTs with larger chiral angles is high, ${ }^{7-15}$ although the origin of this near-armchair preference is the subject of theoretical discussion. ${ }^{16-18}$ During the CVD growth of SWCNTs, carbon cap structures are generated on catalyst nanoparticles, followed by lifting off to elongate the structures into tubes. ${ }^{19,20}$ The measured abundance of SWCNTs with a given chirality is determined by taking the product of the population and average length of each specific type. Since the

${ }^{a}$ Department of Mechanical Engineering, The University of Tokyo, 7-3-1 Hongo, Bunkyo-ku, Tokyo 113-8656, Japan. E-mail: maruyama@photon.t.u-tokyo.ac.jp ${ }^{b}$ National Institute of Advanced Industrial Science and Technology (AIST), 1-2-1 Namiki, Tsukuba, 305-8564, Japan

$\dagger$ Electronic supplementary information (ESI) available. See DOI: $10.1039 / \mathrm{c} 5 \mathrm{ta} 02679 \mathrm{~b}$ energetic stability of the cap structure on the catalyst exhibits a dependence on chirality, ${ }^{\mathbf{8} 16}$ it is possible that cap structures corresponding to certain chiralities are preferentially nucleated, which biases the formation of SWCNT populations of different chiralities. In contrast, the screw dislocation model suggests that chiral SWCNTs with larger chiral angles have higher growth rates ${ }^{17}$ resulting in longer average lengths for near-armchair SWCNTs. The chiral angle dependence of SWCNT growth rates has been studied by in situ Raman measurements of single SWCNT $^{21}$ and SWCNT growth from chirality-separated SWCNT seeds. ${ }^{22}$ As well, a theoretical model of SWCNT abundance considering both nucleation probability and growth rates has been reported. ${ }^{18}$ Despite these efforts, an experimental analysis of the detailed chirality distribution is still required to obtain an improved understanding of chirality-controlled growth.

Since conventional methods used to determine SWCNT chirality distribution cannot distinguish between the contributions of population and length, novel methods to analyse chirality in detail are needed. As an example, spectroscopic evaluation of ensemble SWCNT samples, including SWCNT dispersions in solvent and SWCNT network films, yields chirality abundance data that reflect both populations and lengths..$^{7-9,14}$ In order to investigate SWCNT structures on a oneby-one basis, it is necessary to isolate the SWCNTs. Studies of isolated SWCNTs, however, do not give SWCNT populations as long as the measurements are performed in a random manner. ${ }^{10,11,13,23}$ This occurs because longer SWCNTs tend to appear more frequently in the detection area, and so the count of certain chiralities is affected by both populations and lengths simultaneously. Compared with randomly oriented SWCNTs, aligned SWCNTs lying on substrates represent better candidates for systematic measurements, and SWCNTs both isolated and aligned on substrates have been grown using crystalline substrates $^{24}$ or laminar gas-flow. ${ }^{25}$ Although the chirality 
distributions of horizontally aligned SWCNTs have been studied, ${ }^{12,15,26,27}$ there have been no reports on the independent evaluation of the chirality dependence of both population and length.

In this study, we evaluated SWCNTs by utilizing a horizontally aligned morphology in conjunction with Raman mapping measurements. In this manner, a detailed chirality distribution of SWCNTs was obtained by independently investigating populations and lengths.

\section{Experimental method}

Horizontally aligned SWCNTs were grown on crystal quartz substrates using an alcohol CVD method. ${ }^{28}$ By tuning the growth conditions described in detail in our previous reports, ${ }^{29,30}$ SWCNTs with moderate densities were prepared. Briefly, the growth procedure employed ethanol vapour (partial pressure $\sim 30 \mathrm{~Pa}$ ) together with an $\mathrm{Ar} / \mathrm{H}_{2}(3 \%)$ gas mixture at $750{ }^{\circ} \mathrm{C}$ for $15 \mathrm{~min}$, using an Fe catalyst patterned by photolithography and vacuum deposition (nominal thickness $\sim 0.2 \mathrm{~nm}$ ). To avoid the Raman signal of the crystal quartz substrate, horizontally aligned SWCNTs were subsequently transferred onto $\mathrm{SiO}_{2} / \mathrm{Si}$ substrates using poly(methyl methacrylate) films. ${ }^{31}$ Marker structures were fabricated by photolithography, sputter deposition of Ti, and lift-off to confirm the measurement positions under the optical microscope associated with the Raman measurement system. Raman spectra of fabricated samples were obtained using a Raman spectrometer (Renishaw, inVia) with four excitation wavelengths: $488 \mathrm{~nm}$ $(2.54 \mathrm{eV}), 532 \mathrm{~nm}(2.33 \mathrm{eV}), 633 \mathrm{~nm}(1.96 \mathrm{eV})$, and $785 \mathrm{~nm}$ (1.58 eV). In particular, high-resolution Raman mapping measurements were performed with the excitation at $488 \mathrm{~nm}$. The polarization angle of the excitation laser was adjusted such that it was parallel to the alignment direction of the SWCNTs. The streamline-shaped laser spot was $0.8 \mu \mathrm{m}$ wide and $20 \mu \mathrm{m}$ long, with an intensity of $\sim 10 \mathrm{~mW}$ (power density $\sim 0.6 \mathrm{~mW}$ $\mu \mathrm{m}^{-2}$ ). The acquisition time for one spectrum ranged from 60 to $90 \mathrm{~s}$ and the mapping resolution was $0.6 \mu \mathrm{m}$ in the SWCNT alignment direction and $0.2 \mu \mathrm{m}$ in the direction perpendicular to the alignment. Raman shifts were calibrated using sulfur as a reference. Radial breathing mode (RBM) peaks were fitted with a Lorenz function to determine the RBM frequencies $\left(\omega_{\mathrm{RBM}}\right)$ and the chiralities of the SWCNTs were assigned based on these $\omega_{\mathrm{RBM}}$ values together with the excitation energy, ${ }^{23}$ employing Kataura plots. ${ }^{32,33}$ The lengths of SWCNTs were determined from Raman mapping images and/or by scanning electron microscopy (SEM, Hitachi, S-4800).

\section{Results and discussion}

Fig. 1a shows a SEM image of the horizontally aligned SWCNTs as well as the metal markers on $\mathrm{SiO}_{2} / \mathrm{Si}$ substrate. In this image, SWCNTs with moderate densities $(\sim 2$ tubes per $\mu$ m near the catalyst pattern) are observed. Raman measurements were performed at the four excitation wavelengths, and the resulting spectra and $\omega_{\text {RBM }}$ distributions are presented in Fig. $1 \mathrm{~b}$ and c. The $\omega_{\mathrm{RBM}}$ values, ranging from 100 to $260 \mathrm{~cm}^{-1}$, indicate that the SWCNTs were approximately $0.9-2.0 \mathrm{~nm}$ in diameter, including both metallic and semiconducting SWCNTs. The chiralities of the larger diameter SWCNTs were difficult to assign because their $\omega_{\mathrm{RBM}}$ values were quite close to one another in the low frequency region of the Kataura plot. In this report, when assessing the diameter distributions of the SWCNTs, we considered it appropriate to use the $\omega_{\mathrm{RBM}}$ values in the range of $180-212 \mathrm{~cm}^{-1}$, obtained with the excitation at 488 $\mathrm{nm}$, for chirality assignment.

The samples were carefully investigated by Raman mapping measurements with a $488 \mathrm{~nm}$ excitation wavelength. Measurements were conducted over rectangular areas $\sim 15 \mu \mathrm{m}$ wide in the SWCNT alignment direction and totally $\sim 320 \mu \mathrm{m}$ wide in the perpendicular direction, including the catalyst patterned area in which the SWCNT growth had begun at the centre of the rectangle. These regions contain more than $\sim 1200$ aligned SWCNTs, and 82 RBM peaks with $\omega_{\text {RBM }}$ values ranging from 180 to $212 \mathrm{~cm}^{-1}$ were obtained. The well-organized morphology of the horizontally aligned SWCNTs and the systematic measurement process enabled us to investigate all resonant SWCNTs grown from the catalyst area to the alignment area without multiple counting. Fig. 2a shows typical RBM spectra of a SWCNT acquired at five different points along the tube axis. A $\omega_{\text {RBM }}$ deviation of less than $\pm 1 \mathrm{~cm}^{-1}$ was observed along a nanotube. Fig. 2b presents a SEM image superimposed over Raman mapping that shows the G-band intensities of the aligned SWCNTs. Here the $\omega_{\mathrm{RBM}}$ values of the SWCNTs are indicated by arrows. The RBM spectra in Fig. 2a correspond to the SWCNT circled in blue in Fig. 2b. Lengths of SWCNTs were determined by Raman mapping and SEM observation. When entire SWCNTs fell within Raman mapping areas, SWCNT
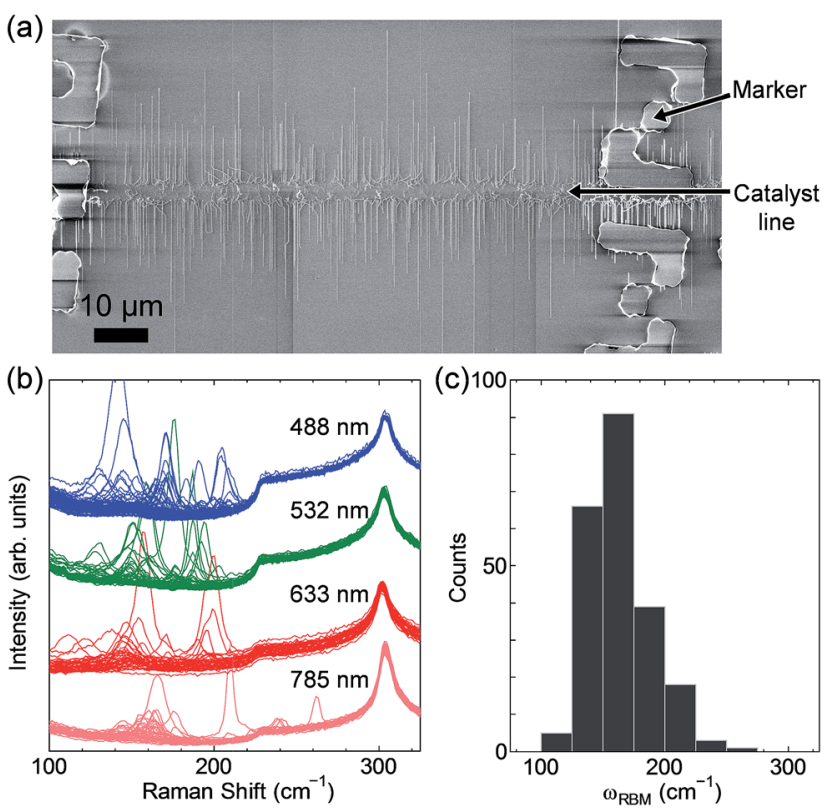

Fig. 1 (a) SEM image of horizontally aligned SWCNTs and marker structures on a $\mathrm{SiO}_{2} / \mathrm{Si}$ substrate. The image is composed of multiple SEM images. (b) Raman spectra of aligned SWCNTs. Excitation wavelengths are 488,532, 633, and $785 \mathrm{~nm}$. (c) Distribution of RBM frequencies. 
lengths were determined solely by Raman mapping by investigating the successive appearance of RBM peaks with similar $\omega_{\text {RBM }}$ values. When the SWCNTs extended beyond the Raman mapping area, the lengths were determined by referring to SEM observations. In this case, the SWCNT density at the edges of Raman mapping areas is much lower than that near the catalyst pattern, and thus we can unambiguously find one corresponding SWCNT in SEM images by comparing Raman mapping images and SEM images. Further details of length determination are shown in Fig. S1 in the ESI. $\dagger$ According to these procedures, we obtained both the $\omega_{\mathrm{RBM}}$ values and the lengths of individual SWCNTs.

Fig. 3a shows the acquired $\omega_{\text {RBM }}$ values together with Kataura plots, ${ }^{33}$ while Fig. $3 \mathrm{~b}$ provides the $\omega_{\mathrm{RBM}}$ distribution. Several Kataura plots have been reported in previous studies. ${ }^{33-37}$ The Kataura plots employed here were originally constructed by the fitting of experimental $\omega_{\text {RBM }}$ values of vertically aligned SWCNTs obtained with a tunable laser excitation wavelength. ${ }^{33}$ As shown in Fig. $3 a$ and $b$, the $\omega_{\text {RBM }}$ values are clearly divided into five distinct regions, believed to correspond to the family for which $2 n+m=29$, including $(10,9),(11,7)$, $(12,5),(13,3)$, and $(14,1)$. These chiralities exhibit third interband transition energies $\left(E^{\mathrm{S}}{ }_{33}\right)$ of $2.56-2.62 \mathrm{eV}$, values of which are within $0.1 \mathrm{eV}$ of the excitation energy of $2.54 \mathrm{eV}(488 \mathrm{~nm})$. The $(12,7)$ chirality is also regarded as a possible candidate since its $\omega_{\mathrm{RBM}}$ value is approximately $183 \mathrm{~cm}^{-1}$; however, its $E_{33}^{\mathrm{S}}$ of $2.71 \mathrm{eV}$ is too far from the excitation energy. Additionally, the family for which $2 n+m=32$, having $E_{33}^{\mathrm{S}}$ values of $2.42-2.44 \mathrm{eV}$, is an alternative choice for the $\omega_{\mathrm{RBM}}$ range of $180-195 \mathrm{~cm}^{-1}$. To confirm these assignments, we investigated the same area of the sample by Raman measurements using a $532 \mathrm{~nm}$ excitation wavelength. In this case, corresponding RBM peaks were not found in the range of $180-195 \mathrm{~cm}^{-1}$ at the points where peaks had appeared in response to the $488 \mathrm{~nm}$ excitation. Thus, we excluded the possibility that the peaks in the range of 180-195 $\mathrm{cm}^{-1}$ resulting from the $488 \mathrm{~nm}$ excitation were from the $2 n+m=32$ family. We therefore assigned the SWCNTs with $\omega_{\text {RBM }}$ values of 180.9-184.2, 190.0-193.1, 197.4-200.7, 202.5-205.2, and 207.7-211.7 to chiralities (10,9), (11,7), (12,5),
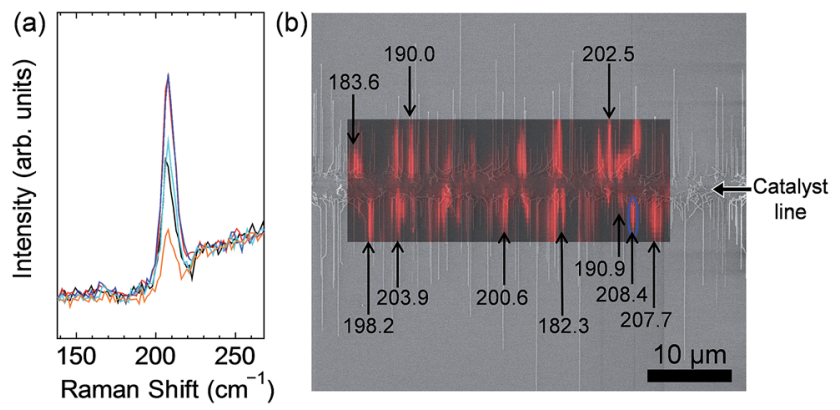

Fig. 2 (a) Raman spectra of the same SWCNT measured at different five points along its axis. Excitation wavelength is $488 \mathrm{~nm}$. (b) Superimposed SEM and Raman mapping images. The red contrast of the Raman mapping image shows the G-band intensity obtained with a $488 \mathrm{~nm}$ excitation. The values indicated by arrows are the RBM frequencies $\left(\mathrm{cm}^{-1}\right)$ of the SWCNTs. Raman spectra shown in (a) are from the SWCNT in the blue circle.
$(13,3)$, and $(14,1)$, respectively. The $\omega_{\text {RBM }}$ values of SWCNTs of a given chirality were found to vary by approximately $\pm 2 \mathrm{~cm}^{-1}$, likely as a result of variations in the environmental conditions of the SWCNTs. The $\omega_{\mathrm{RBM}}$ values of SWCNTs are known to be affected by interactions with the surrounding environment, ${ }^{35}$ including underlying substrates, ${ }^{38}$ other SWCNTs forming bundles, ${ }^{\mathbf{3 9 , 4 0}}$ and adsorbed water molecules. ${ }^{\mathbf{4 0 , 4 1}}$ The $\omega_{\mathrm{RBM}}$ values were plotted against the diameter of assigned chiralities in comparison with the previous reports (Fig. S2 $\dagger$ ). We note that the assigned five chiralities do not represent all of the grown chiralities, but we focused on the chiralities due to measurement limitations.

Subsequently, the chiral angle dependence of the SWCNT growth was assessed using the obtained chirality-resolved SWCNT populations. Although the assigned five species of SWCNTs had different diameters as well as chiral angles, we considered that their diameter range of 1.14-1.29 nm was sufficiently small so as to have a minimal effect on the SWCNT growth. The SWCNT populations of specific chiralities were thus plotted against the chiral angles (Fig. 4a). These data show a relatively small population of the $(10,9)$ SWCNTs, presumably due to the chirality dependence of the RBM intensity, since the RBM intensity is known to be weaker for SWCNTs with larger diameters and larger chiral angles. ${ }^{42}$ It is reasonable to assume that the RBM intensity did not affect the measurement results in this study because the Raman spectra of individual SWCNTs were acquired and the number of RBM peaks was counted on a one-by-one basis. However, we cannot exclude the possibility that RBM peaks weaker than the threshold value that was used to assess the presence of peaks were neglected. Fig. S3† shows the RBM intensity, normalized by the Si peak intensity at $\sim 303$
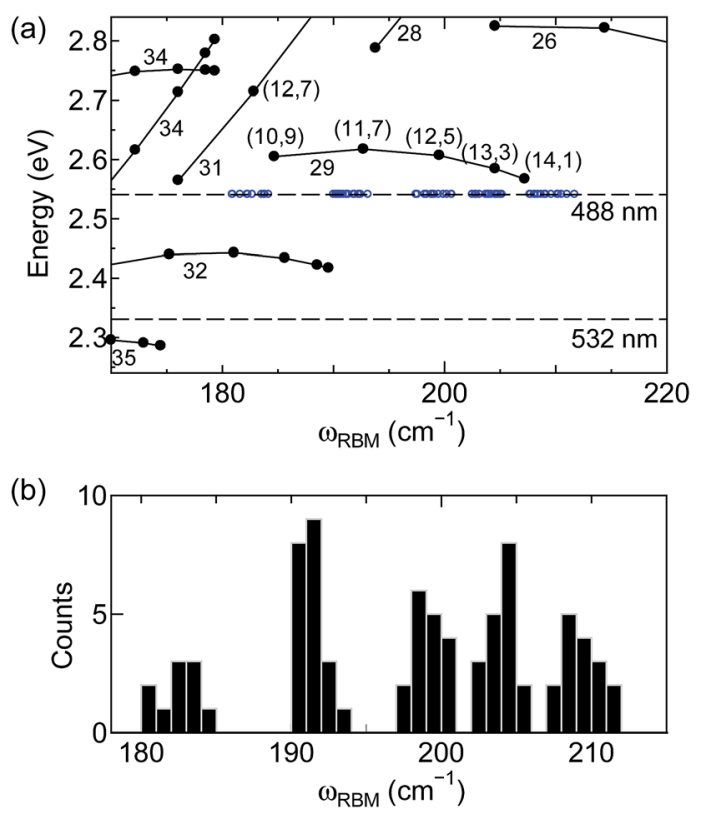

Fig. 3 (a) Transition energies versus RBM frequencies. Blue open circles are acquired values from individual SWCNTs with a $488 \mathrm{~nm}$ excitation. Black dots are calculated Kataura plots. ${ }^{33}$ Chiral indices $(n, m)$ and $2 n+m$ family numbers are denoted. (b) Distribution of RBM frequencies for 82 different SWCNTs. 
$\mathrm{cm}^{-1}$, as a function of $\omega_{\mathrm{RBM}}$. A comparison of the RBM intensities at different wavenumbers demonstrates that the intensity tends to be stronger in the higher wavenumber regions. We believe that all the SWCNTs, with the exception of the $(10,9)$ species, had sufficient peak intensity and thus all the SWCNT chiralities were observed. Therefore the populations derived for the SWCNTs grown in this study are considered to have been unbiased by chiral angles.

We then investigated the effect of the chiral angle on SWCNT lengths. Fig. 4 b shows the average lengths of SWCNTs having different chiralities as functions of the chiral angle. Here, the average SWCNT length over the five chiralities was in the range of 3-4 $\mu \mathrm{m}$. This result indicates that the chiral angle did not have a pronounced effect on the SWCNT lengths under the present growth conditions. These findings are important since the evaluation of the lengths of chiralityresolved SWCNTs is difficult using conventional methods, and there have been only a few reports of such evaluations to date. ${ }^{22,43}$

The data herein demonstrate that both the populations and lengths of SWCNTs exhibit no clear dependence on the chiral angle. We consider that the growth of SWCNTs with a diameter of 1.14-1.29 nm in this study was unaffected by the chiral angle. This is in accordance with previous reports which showed that SWCNTs with a relatively large diameter $(>\sim 1.0 \mathrm{~nm})$ have no chiral angle dependence..$^{\mathbf{8 , 9 4 4 , 4 5}}$ Additionally, the growth of SWCNTs from SWCNT seeds of specific chiralities has shown that SWCNTs with larger chiral angles exhibit higher growth rates but shorter growth lifetimes, resulting in no clear dependence of the final SWCNT lengths on chirality. ${ }^{22}$ It is possible that the SWCNT growth in this study had a similar tendency, and accordingly chiral angle dependence was not directly observed for the SWCNT lengths.

Due to the restricted Raman resonance conditions and the difficulty in assigning larger diameter SWCNTs, the number of assigned chiralities in the present study was limited. However, the data did indicate a relatively flat distribution of populations and uniformity in the lengths of SWCNTs with several different chiralities, which is otherwise difficult to acquire by conventional measurement methods. The application of this technique to SWCNTs obtained under other growth conditions should enable a more detailed study of chirality-controlled SWCNT growth.
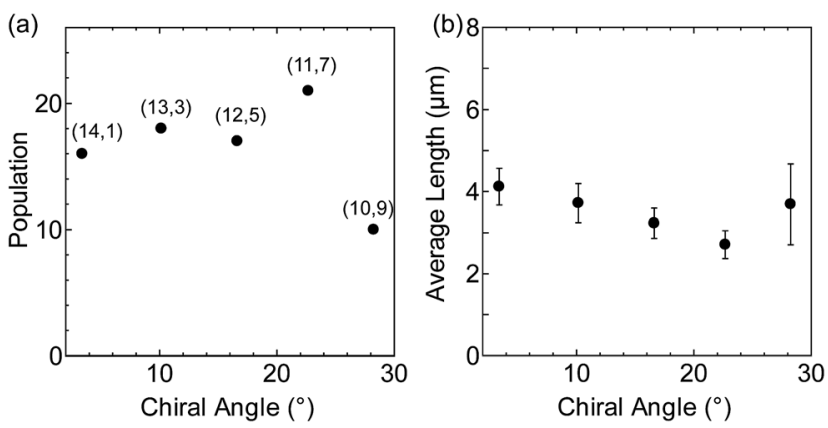

Fig. 4 (a) Populations and (b) average lengths of SWCNTs of five different chiralities plotted against the chiral angle.

\section{Conclusions}

We examined the chiralities of SWCNTs by Raman mapping measurements of horizontally aligned SWCNTs. Through analysing the chirality of individual SWCNTs, we independently evaluated both the populations and lengths of five chiralities in the $2 n+m=29$ family. Neither the chirality-resolved populations nor the lengths of the SWCNTs showed a clear dependence on the chiral angle. This measurement strategy will assist in the future elucidation of the mechanism of the structure-controlled growth of SWCNTs.

\section{Acknowledgements}

Part of this work was financially supported by Grants-in-Aid for Scientific Research (nos 22226006, 25630063, 25107002, 15H02219) and the IRENA Project of JST-EC DG RTD, Strategic International Collaborative Research Program (SICORP). Part of this work was supported by the "Nanotechnology Platform Japan" of the Ministry of Education, Culture, Sports, Science and Technology (MEXT), in Takeda Cleanroom with the help of the Nanofabrication Platform Center of the VLSI Design and Education Center (VDEC), the University of Tokyo, Japan. Part of this work was also supported by Laser Alliance, the University of Tokyo. T.I. was financially supported by a JSPS Fellowship (23-8717).

\section{References}

1 S. Iijima and T. Ichihashi, Nature, 1993, 363, 603-605.

2 N. Hamada, S. Sawada and A. Oshiyama, Phys. Rev. Lett., 1992, 68, 1579-1581.

3 R. Saito, M. Fujita, G. Dresselhaus and M. S. Dresselhaus, Appl. Phys. Lett., 1992, 60, 2204-2206.

4 M. S. Arnold, A. A. Green, J. F. Hulvat, S. I. Stupp and M. C. Hersam, Nat. Nanotechnol., 2006, 1, 60-65.

5 H. Liu, D. Nishide, T. Tanaka and H. Kataura, Nat. Commun., 2011, 2, 309.

6 Y. Li, W. Kim, Y. Zhang, M. Rolandi, D. Wang and H. Dai, J. Phys. Chem. B, 2001, 105, 11424-11431.

7 S. M. Bachilo, L. Balzano, J. E. Herrera, F. Pompeo, D. E. Resasco and R. B. Weisman, J. Am. Chem. Soc., 2003, 125, 11186-11187.

8 Y. Miyauchi, S. Chiashi, Y. Murakami, Y. Hayashida and S. Maruyama, Chem. Phys. Lett., 2004, 387, 198-203.

9 A. Jorio, C. Fantini, M. Pimenta, R. Capaz, G. Samsonidze, G. Dresselhaus, M. S. Dresselhaus, J. Jiang, N. Kobayashi, A. Grüneis and R. Saito, Phys. Rev. B: Condens. Matter Mater. Phys., 2005, 71, 075401.

10 Z. Liu, Q. Zhang and L.-C. Qin, Phys. Rev. B: Condens. Matter Mater. Phys., 2005, 71, 245413.

11 B. Liu, W. Ren, S. Li, C. Liu and H.-M. Cheng, Chem. Commun., 2012, 48, 2409-2411.

12 R. Arenal, P. Löthman, M. Picher, T. Than, M. Paillet and V. Jourdain, J. Phys. Chem. C, 2012, 116, 14103-14107.

13 M. He, H. Jiang, E. I. Kauppinen and J. Lehtonen, Nanoscale, 2012, 4, 7394-7398. 
14 H. Wang, L. Wei, F. Ren, Q. Wang, L. D. Pfefferle, G. L. Haller and Y. Chen, ACS Nano, 2013, 7, 614-626.

15 K. Liu, X. Hong, Q. Zhou, C. Jin, J. Li, W. Zhou, J. Liu, E. Wang, A. Zettl and F. Wang, Nat. Nanotechnol., 2013, 8, 917-922.

16 S. Reich, L. Li and J. Robertson, Chem. Phys. Lett., 2006, 421, 469-472.

17 F. Ding, A. R. Harutyunyan and B. I. Yakobson, Proc. Natl. Acad. Sci. U. S. A., 2009, 106, 2506-2509.

18 V. I. Artyukhov, E. S. Penev and B. I. Yakobson, Nat. Commun., 2014, 5, 4892.

19 Y. Shibuta and S. Maruyama, Chem. Phys. Lett., 2003, 382, 381-386.

20 S. Hofmann, R. Sharma, C. Ducati, G. Du, C. Mattevi, C. Cepek, M. Cantoro, S. Pisana, A. Parvez, F. CervantesSodi, A. C. Ferrari, R. Dunin-Borkowski, S. Lizzit, L. Petaccia, A. Goldoni and J. Robertson, Nano Lett., 2007, 7, 602-608.

21 R. Rao, D. Liptak, T. Cherukuri, B. I. Yakobson and B. Maruyama, Nat. Mater., 2012, 11, 213-216.

22 B. Liu, J. Liu, X. Tu, J. Zhang, M. Zheng and C. Zhou, Nano Lett., 2013, 13, 4416-4421.

23 A. Jorio, R. Saito, J. H. Hafner, C. Lieber, M. Hunter, T. McClure, G. Dresselhaus and M. S. Dresselhaus, Phys. Rev. Lett., 2001, 86, 1118-1121.

24 C. Kocabas, S.-H. Hur, A. Gaur, M. A. Meitl, M. Shim and J. A. Rogers, Small, 2005, 1, 1110-1116.

25 S. Huang, X. Cai and J. Liu, J. Am. Chem. Soc., 2003, 125, 5636-5637.

26 L. Zhang, Z. Jia, L. Huang, S. O’Brien and Z. Yu, J. Phys. Chem. C, 2007, 111, 11240-11245.

27 H. Chu, J. Wang, L. Ding, D. Yuan, Y. Zhang, J. Liu and Y. Li, J. Am. Chem. Soc., 2009, 131, 14310-14316.

28 S. Maruyama, R. Kojima, Y. Miyauchi, S. Chiashi and M. Kohno, Chem. Phys. Lett., 2002, 360, 229-234.

29 S. Chiashi, H. Okabe, T. Inoue, J. Shiomi, T. Sato, S. Kono, M. Terasawa and S. Maruyama, J. Phys. Chem. C, 2012, 116, 6805-6808.
30 T. Inoue, D. Hasegawa, S. Badar, S. Aikawa, S. Chiashi and S. Maruyama, J. Phys. Chem. C, 2013, 117, 11804-11810.

31 L. Jiao, B. Fan, X. Xian, Z. Wu, J. Zhang and Z. Liu, J. Am. Chem. Soc., 2008, 130, 12612-12613.

32 H. Kataura, Y. Kumazawa, Y. Maniwa and I. Umezu, Synth. Met., 1999, 103, 2555-2558.

33 P. T. Araujo, S. K. Doorn, S. Kilina, S. Tretiak, E. Einarsson, S. Maruyama, H. Chacham, M. Pimenta and A. Jorio, Phys. Rev. Lett., 2007, 98, 067401.

34 C. Fantini, A. Jorio, M. Souza, M. S. Strano, M. S. Dresselhaus and M. Pimenta, Phys. Rev. Lett., 2004, 93, 147406.

35 P. T. Araujo, I. Maciel, P. Pesce, M. Pimenta, S. K. Doorn, H. Qian, A. Hartschuh, M. Steiner, L. Grigorian, K. Hata and A. Jorio, Phys. Rev. B: Condens. Matter Mater. Phys., 2008, 77, 241403.

36 J. S. Soares, L. G. Cançado, E. B. Barros and A. Jorio, Phys. Status Solidi B, 2010, 247, 2835-2837.

37 J. Wang, J. Yang, D. Zhang and Y. Li, J. Phys. Chem. C, 2012, 116, 23826-23832.

38 Y. Zhang, J. Zhang, H. Son, J. Kong and Z. Liu, J. Am. Chem. Soc., 2005, 127, 17156-17157.

39 P. T. Araujo, C. Fantini, M. M. Lucchese, M. S. Dresselhaus and A. Jorio, Appl. Phys. Lett., 2009, 95, 261902.

40 S. Chiashi, K. Kono, D. Matsumoto, J. Shitaba, N. Homma, A. Beniya, T. Yamamoto and Y. Homma, Phys. Rev. B: Condens. Matter Mater. Phys., 2015, 91, 155415.

41 Y. Homma, S. Chiashi, T. Yamamoto, K. Kono, D. Matsumoto, J. Shitaba and S. Sato, Phys. Rev. Lett., 2013, 110, 157402.

42 V. N. Popov, L. Henrard and P. Lambin, Nano Lett., 2004, 4, 1795-1799.

43 J. P. Casey, S. M. Bachilo, C. H. Moran and R. B. Weisman, ACS Nano, 2008, 2, 1738-1746.

44 H. Ago, S. Imamura, T. Okazaki, T. Saito, M. Yumura and M. Tsuji, J. Phys. Chem. B, 2005, 109, 10035-10041.

45 Y. Oyama, R. Saito, K. Sato, J. Jiang, G. G. Samsonidze, A. Grüneis, Y. Miyauchi, S. Maruyama, A. Jorio, G. Dresselhaus and M. S. Dresselhaus, Carbon, 2006, 44, 873-879. 